\title{
Grief Process and the COVID-19 Pandemic: Wise Intervention in Vulnerable Groups and Survivors
}

\author{
Bita Vahdani ${ }^{1}$, Seyyed Mohammad Hossein Javadi (iD ${ }^{2,}{ }^{*}$, Mohammad Sabzi Khoshnami (iD) ${ }^{3}$ and \\ Moslem Arian (iD ${ }^{3}$ \\ ${ }^{1}$ Department of Psychiatry, Qazvin University of Medical Sciences, Qazvin, Iran \\ ${ }^{2}$ Social Work Department, University of Social Welfare and Rehabilitation Sciences, Tehran, Iran \\ ${ }^{3}$ Department of counseling, University of Social Welfare and Rehabilitation Sciences, Tehran, Iran \\ "Corresponding author: Social Work Department, University of Social Welfare and Rehabilitation Sciences, Tehran, Iran. Email: javadismh4@gmail.com \\ Received 2020 April 18; Revised 2020 April 25; Accepted 2020 May 01.
}

Keywords: Complicated Grief, COVID-19, Grief Process, Iran, Psychosocial Interventions

\section{Dear editor,}

The global COVID-19 pandemic has dramatically attacked our planet, and the entire human race faces great distress. The novel Coronavirus has changed everyday life around the world quite suddenly, often in unexpected and unavoidable ways. The number of confirmed cases and deaths reported by WHO as of April 23, 2020, is 2,628,143 and 183,090, respectively. In Iran, 85,996 cases have tested positive for COVID-19, with 5,391 deaths since the onset of the pandemic. The deadly threat of coronavirus can be felt in villages, rural communities, cities, and suburbs all over the world.

On an individual level, one of the challenges of living in this situation is dealing with initiation or exacerbation of anxiety and non-specific mental problems (e.g., mood problems, sleep disturbance, trauma-based disorders, panic-like symptoms, substance abuse), as well as coping with death, dying and grief issues. In many countries, based on cultural and religious traditions, there are some group rituals that can help people to self-soothe, calm themselves, and find a sense of peace after the loss of a loved one. They are powerful tools that can facilitate the grief process and the adjustment to bereavement. But conducting funerals amid the coronavirus outbreak has been "heartbreaking" as restrictions have meant only 10 people could attend. Everywhere the coronavirus has struck, regardless of culture or religion, ancient rituals to honor the dead and comfort the bereaved have been cut short or abandoned for fear of spreading it further. So conducting a funeral ritual and gathering with family members and close friends are no longer options in these times.

Here some critical questions can be raised: What happens when the traditional rituals are taken away? What happens when a loved one is dying, and the family is unable to accompany her/him during the last hours and also the burying process? Many deaths have already happened, and many more will occur definitely and death in quarantine leaves nowhere to grieve. Finding clear answers is not an easy and straightforward task for the grief therapist, caregiver, counselor, or psychiatrist. There are no established protocols or guidelines or predetermined solutions to the problem As if we need a novel solution for this novel problem.

For the bereaved families, grief reaction would be a continuum that involves societies and depends on different cultural, religious, socioeconomic, and educational factors (1). Grief is an unwelcome but inevitable event in our lives, so we have no choice except to live through it.

It seems that grief becomes increasingly prolonged, complicated, or even traumatic when the normal rituals and the traditional funeral concerning death do not take place. Complicated grief may be defined through maladaptive thoughts, feelings, emotions, and behaviors that make grief even more intense and disabling (2). In this dramatic situation, appropriate leadership, and structure, and clear delineation of duties and responsibilities are critical (3). Moreover, developing a safety plan and communication strategy with an appropriate intervention will assist the survivors and family members to overcome their grief in a comfortable and empathetic environment. 
In Iran, from the onset of coronavirus outbreak, the Ministry of Health, Treatment and Medical Education have implemented widely accessible hotlines for psychosocial support that could be reached through basic tools in social media, computer and telephone follow-ups. They were meant to help the medical system on screening of the patients, the detection of the infection, and also giving the people psychological consultation to evaluate the level of mental health and to improve the coping mechanisms of the general population.

A psychological intervention group has been selected and trained to intervene in this crisis. Specialists in this group have been trained to establish a supportive rapport, to be active listeners, and to challenge thought distortions, anxiety, and distress that can contribute to guilt feelings (4). They also teach mindfulness-based techniques, progressive muscle relaxation, and guided imagery to decrease the stress level (5).

In such a situation, grief and loss of online counseling should be prepared for the bereaved family members. Given that a large number of people have endured the loss of loved ones, bereavement support groups for surviving relatives will also play a therapeutic role.

At the communal level, psychosocial specialists working through the pandemic should focus on managing the emotional alliance with the survivors. They are recommended to pay specific attention to the concerns of the public while providing exact useful information and reducing the gap between public attitudes and epidemiological facts and extend their support during the follow-up (6). In Iran, for reaching this goal, the educated and expert social workers around the country try to connect with the patients' families in the hospitals, identify and prepare to address the psychosocial needs of families' and survivors in dealing with grief, anxiety, anger, loss of financial support, posttraumatic stress, and public stigma. In cooperation with other organizations such as state welfare, the Red Crescent, non-governmental organizations, the case management process will facilitate healing and resilience by encouraging individuals, families, and communities to actively engage.

In the COVID-19 pandemic, the responsibility of mental health professionals will be careful assessment, construction of new hopes and dreams in survivors, and preparation of mental health services for pre-existing chronic disorders. Psychiatrists may provide grief and loss counseling for bereaved relatives to improve the sense of social supports and to reduce the distress and burden of trauma. In the long term aftermath, pharmacologic intervention, trauma-focused therapies and family resilience-oriented approach (7) may be applied as inevitable measures, especially for some specific groups with the previous history of mental disorders, and for the survivors with suicidal ideas and impairment in functioning. For patients who are unable to access their usual mental health providers, telepsychiatry or online psychological interventions can be a helpful alternative during the pandemic $(8,9)$.

In crisis management, health policymakers probably pay less attention to the establishment of psychosocial supports for specific groups of the population. At the same time, the governments may be concerned with the potential obstacles imposed by the pandemic in health care providers such as, contact barriers which enable clients to read facial and nonverbal expressions from masked faces, lack of protective equipment, difficulty accessing telephone numbers, and addresses of bereaved families, providing financial resources, and medication needs.

Finally, the preparation of these matters can help societies to establish more appropriate and effective psychosocial services for vulnerable people at the time of the pandemic.

\section{Footnotes}

Authors' Contribution: Bita Vahdani and Seyyed Mohammad Hossein Javadi designed the study and confirmed the scientific content. Moslem Arian and Mohammad Sabzi Khoshnami collected the data and revised the manuscript. All the authors read and approved the final manuscript

Conflict of Interests: Authors declared no conflict of interest.

Funding/Support: This article is a letter to the editor, and no financial support was received from any governmental or academic organizations.

\section{References}

1. Shear MK, Reynolds III CF, Simon NM, Zisook S, Stein MB. Complicated grief in adults: Treatment. In: Post TW, editor. . Waltham: UpToDate Inc; 2018.

2. Shear MK, Reynolds III CF, Simon NM, Zisook S, Stein MB. Grief and bereavement in adults: clinical features. In: Post TW, editor. . Waltham: UpToDate Inc; 2018.

3. Huremović D. Psychiatry of Pandemics: A Mental Health Response to Infection Outbreak. North Shore University Hospital Manhasset, NY, USA; 2019

4. Good Therapy. Survivor guilt. 2018, [cited 2019 January 11]. Available from: https://www.goodtherapy.org/blog/psychpedia/survivorguilt. 
5. Havice-Cover PJ, Drennen C. Pandemic influenza: quaran- tine, isolation and social distancing. Colorado: The Colorado Department of Human Services Division of Mental Health; 2020. Available from: http://www.realisticpreparedness.com/downloads/ PanFluQuarantineIsolation.pdf.

6. Manderscheid RW. Preparing for pandemic Avian influenza: ensuring mental health services and mitigating panic. Arch Psychiatr Nurs. 2007;21(1):64-7. doi:10.1016/j.apnu.2006.10.003. [PubMed:17258112].

7. Walsh F. Traumatic loss and major disasters: strengthening fam- ily and community resilience. Fam Process. 2007;46(2):207-27. doi: 10.1111/j.1545-5300.2007.00205.x. [PubMed: 17593886].

8. Zarghami M. Psychiatric Aspects of Coronavirus (2019-nCoV) Infection. Iranian Journal of Psychiatry and Behavioral Sciences. 2020;14(1). doi: 10.5812/ijpbs.102957.

9. Javadi SMH, Arian M, Qorbani-Vanajemi M. The Need for Psychosocial Interventions to Manage the Coronavirus Crisis. Iranian Journal of Psychiatry and Behavioral Sciences. 2020;14(1). doi: 10.5812/ijpbs.102546. 